\title{
Integrated Optimization of Pipe Routing and Clamp Layout for Aeroengine Using Improved MOALO
}

\author{
Qiang Liu, ${ }^{1}$ Zhi Tang $\mathbb{D}^{1}{ }^{1}$ Huijuan Liu, ${ }^{1}$ Jiapeng $\mathrm{Yu}^{2}{ }^{2}$ Hui $\mathrm{Ma}^{2}$ and Yonghua Yang ${ }^{3}$ \\ ${ }^{1}$ School of Information and Control Engineering, Liaoning Petrochemical University, Fushun 113001, China \\ ${ }^{2}$ School of Mechanical Engineering and Automation, Northeastern University, Shenyang 110819, China \\ ${ }^{3}$ AECC Shenyang Engine Design Institute, Shenyang 110000, China
}

Correspondence should be addressed to Zhi Tang; 1036756484@qq.com

Received 23 November 2020; Revised 20 June 2021; Accepted 2 July 2021; Published 28 July 2021

Academic Editor: Angelo Cervone

Copyright (c) 2021 Qiang Liu et al. This is an open access article distributed under the Creative Commons Attribution License, which permits unrestricted use, distribution, and reproduction in any medium, provided the original work is properly cited.

Pipe routing and clamp layout for aeroengine are NP-hard computational problems and complex engineering design processes. Besides space constraints and engineering rules, there are assembly constraints between pipes and clamps, which usually lead to repeatedly modifications between pipe routing and clamp layout designs. In order to solve the problems of assembly constraints and design coupling between them, an integrated optimization method for pipe routing and clamp layout is proposed. To this end, the MOALO (multiobjective ant lion optimizer) algorithm is modified by introducing the levy flight strategy to improve the global search performance and convergence speed, and it is further used as a basic computation tool. The integrated optimization method takes pipe and clamp as a whole system and then solves the Pareto solution set of pipe-clamp layouts by using improved MOALO, where the pipe path, clamp position, and rotation angle are selected as decision variables and are further optimized. Inspired by engineering experience, a clamp-based pipe path mechanism considering regular nodes is established to deal with assembly constraint problem. The proposed method comprehensively considers engineering rules of pipe routing and clamp layout and realizes the overall layout optimization of pipe-clamp system while guaranteeing the assembly constraints between pipes and clamps. Finally, some numerical computations and routing examples are conducted to demonstrate the feasibility and effectiveness of the proposed method.

\section{Introduction}

The pipe system of aeroengine consists of a series of pipes fixed by clamps. As an important part of aeroengine, the quality and efficiency of pipe routing design have an important impact on the reliability and design cycle of the whole product. In the aspect of automatic pipe routing design, many scholars have carried out extensive research and put forward a series of optimization algorithms for automatic pipe routing, such as maze algorithm [1], heuristic algorithm $[2,3], A *$ algorithm [4], graph-based routing algorithms [5], branch pipe routing based on local twice learning probabilistic roadmap method (LTL-PRM) [6], and routing method based on intelligent optimization [7-14]. With the development of multiobjective optimization algorithm, some scholars began to explore the Pareto solution method of multiobjective pipe routing in recent years. Sui and others proposed an improved NSGA-II algorithm to find the best ship pipe [15]. Based on Kriging model and NSGA-II algorithm, Liu proposed a multiobjective pipe routing method with pipe length, smoothness, and natural frequency as optimization objectives [16]. Ahmed and others applied the NSGA-II algorithm to study the multiobjective path planning problem in twodimensional (2D) space [17]. However, pipe routing involves a series of optimization objectives and constraints such as space and rules; the efficient routing algorithm and system still remain an open area.

In a pipe system, the clamp plays a supporting and fixing role on the pipe and has an important impact on the vibration performance and stability of the pipe. It is one of the important research directions to optimize the vibration performance of pipes by adjusting the position of clamps. Many references provide useful exploration for the arrangement of clamps [18-22]. Due to the assembly constraint relationship 
between pipes and clamps, pipe routing and the clamp layout design further affect each other. Most existing research on pipe routing and clamp layout are performed independently, and the assembly constraints and design coupling relationship between them are rarely considered, which often leads to repeated modifications.

The pipe routing design is a classic $3 \mathrm{D}$ path planning problem with spatial constraints, which is a NP-hard computational problem. On the other hand, clamp layout is a optimization problem that needs to determine its installation location, which can be formulated as a classical location problem; in addition, it needs to determine its rotation angle, which is a pose optimization problem. Due to the assembly constraints between pipes and clamps, pipe routing and clamp layout designs are coupled with each other, which makes the design be more complex and leads to repeated modifications.

To deal with the coupling relationship between pipe routing and clamp layout, this paper treats them as a whole to optimize. The study is aimed at presenting a routing method that automatically generates pipe routing while considering clamp constraints, with respect to a series of engineering rules. The reminder of this paper is arranged as follows. Section 2 gives the problem description, engineering rules, and space modeling method. Section 3 proposed an improved MOALO by introducing levy flight strategy, which is further used to solve the pipe-clamp layout problem. Section 4 presents a multiobjective routing method to seek the optimal pipe and clamp layouts simultaneously by using improved MOALO, where the clamp-based pipe path mode, individual encoding design, objective function computations, constraints processing, routing system, and algorithm flowchart are discussed in detail. Section 5 performs some routing examples. Section 6 finally concludes this paper.

\section{Problem Description and Spatial Modeling}

2.1. Optimization Objectives and Constraints of Pipe Routing. As the core part of pipe system design, pipe routing mainly considers but is not limited to the following objectives and constraints:

Objective 1: the total length of pipe should be as short as possible.

Objective 2: the pipe path should be as smooth as possible to reduce flow resistance.

Constraint 1: the pipe should avoid obstacles and some specific areas.

Constraint 2: the pipe should bypass the accessories to avoid crossing.

Constraint 3: the bending angle of pipe should not be less than 90 .

Constraint 4: the pipe should be arranged close to the casing surface to obtain better vibration and save space.

Constraint 5: the bending radius should match the pipe diameter.

Constraint 6: minimum length requirement of pipe straight section to meet manufacturability requirement.

2.2. Optimization Objectives and Constraints of Clamp Layout. The clamp is an important part of the aeroengine

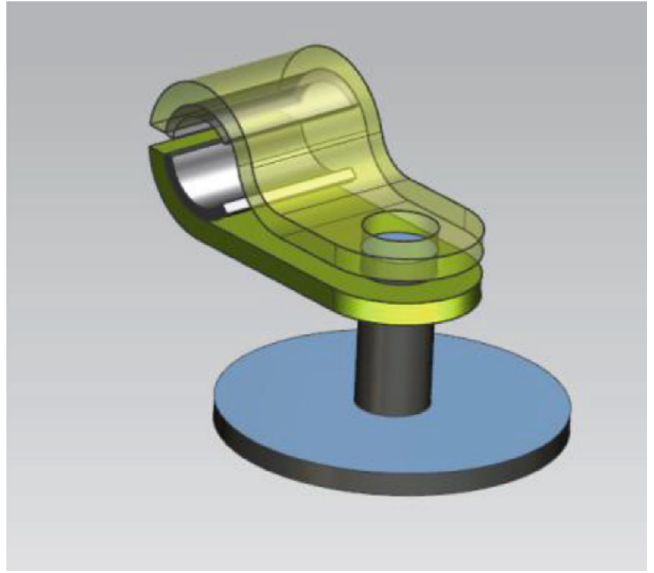

Figure 1: A simplified clamp CAD model.

pipe system, which usually includes three types: the clamp based on mounting, the clamp based on mounting edge, and double-clamp, which plays a supporting and stabilizing role on the pipe. The type, size, and shape of the clamp are relatively fixed (although its supporting parts such as mounting base and bracket allow a certain range of size changes), which is different from the free configuration of the pipe. Figure 1 shows a simplified CAD simulation model of the clamp based on the mounting.

In terms of clamp arrangement, the following constraints are mainly considered:

Constraint 1: the clamp must be arranged in the designated area.

Constraint 2: according to engineering design experience, if the pipe exceeds a certain length $L_{s}$, clamps should be installed to fix the pipe to improve the stability and vibration performance.

Constraint 3: the casing-clamp-pipe should conform to the assembly constraint relationship.

\subsection{Coupling Relationship between Pipe Routing and Clamp} Layout. The design process of pipe routing and clamp layout influence each other. Generally, the clamp should be arranged first, and then, the pipe should be designed based on the clamp. Due to the clamp can only be arranged in the designated area, and the shape and size of the clamp are relatively fixed, it is difficult to ensure that the clamp-pipecasing meets the assembly constraints if the pipe is routed first. Obviously, the position of the clamp has an intuitive and important impact on the pipe path under the pipe routing based on the clamp. At the same time, when arranging the clamps, it is necessary to predict the general direction of the pipe in advance, so as to make the layout of the clamps in the best position, that is, the pipe direction also has an impact on the layout of the clamps.

Therefore, the pipe and clamp have assembly constraints, they interact with each other in design, and they have coupling relationship. Designers often need to modify many times according to experience to complete decoupling optimization. 


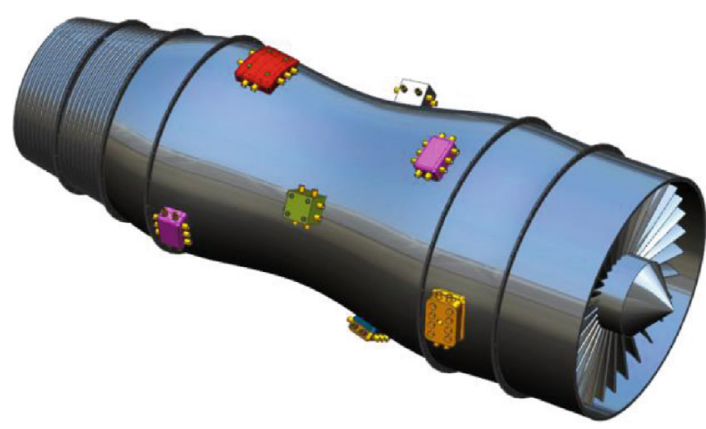

Figure 2: The simplified CAD model.

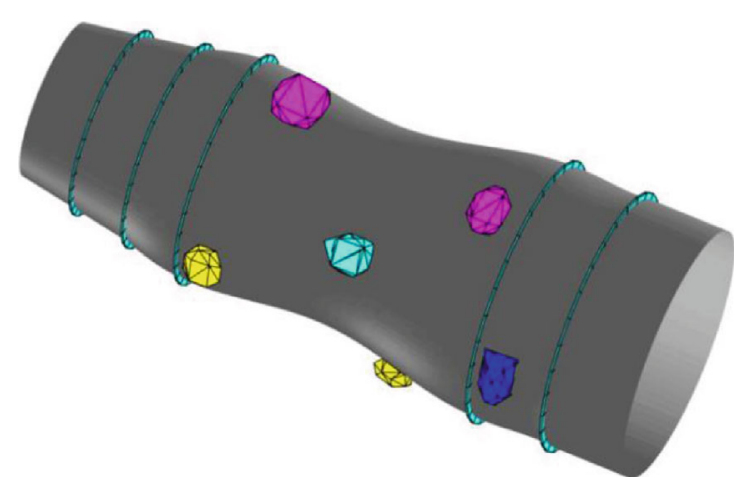

Figure 3: Obstacle model by convex hull in MATLAB.

2.4. Routing Space and Preprocessing. A series of space constraints are included in the aeroengine. Figure 2 shows the simplified CAD model of aeroengine pipe routing simulation. In the aspect of generatrix equation, Siemens/GRIP secondary development program can be used to extract some coordinate points on a certain generatrix on the casing surface, and then, the least square method is used to fit them. In terms of spatial geometry information and accessory obstacles, the grid method can be used to scan, and the convex hull model can be used to model and express the obstacle in MATLAB (as shown in Figure 3). This geometric information will be stored as input information of the algorithm.

\section{Improved Multiobjective Ant Lion Optimizer (IMOALO)}

3.1. MOALO Algorithm. The multiobjective ant lion optimizer [23] is a multiobjective version of ALO algorithm [24]. In order to mimic and model the interaction between ant lion and ant in the trap, ants are required to move over the search space, and antlions are allowed to hunt them and become fitter using traps. Since ants move stochastically in nature when searching for food, the mathematical expression of movement as follows:

$$
\begin{aligned}
X(t)= & {\left[0, \operatorname{cumsum}\left(2 r\left(t_{1}\right)-1\right), \operatorname{cumsum}\left(2 r\left(t_{2}\right)-1\right), \cdots,\right.} \\
& \left.\operatorname{cumsum}\left(2 r\left(t_{n}\right)-1\right)\right],
\end{aligned}
$$

where cumsum is the cumulative sum, $n$ is the maximum number of iterations, $t$ is the current number of iterations, and $r(t)$ is a stochastic function, which can be expressed by Equation (2).

$$
r(t)=\left\{\begin{array}{l}
1, \text { rand }>0.5 \\
0, \text { rand } \leq 0.5
\end{array}\right.
$$

Since each search space has a boundary (variable range), Equation (1) cannot be directly used to update the position of ants. In order to keep the random walks in the search space, the following equation is used to normalize them:

$$
X_{i}^{t}=\frac{\left(X_{i}^{t}-a_{i}\right) \times\left(d_{i}-c_{i}^{t}\right)}{\left(d_{i}^{t}-a_{i}\right)}+c_{i}^{t},
$$

where $d_{i}$ and $a_{i}$ represent the upper and lower bounds of the $i$ th variable, respectively; $d_{i}{ }^{t}$ and $c_{i}{ }^{t}$ represent the upper and lower bounds of the $i$ th variable at $t$ th iteration, which can be represented by Equation (4) and Equation (5):

$$
\begin{aligned}
& c_{i}^{t}=\text { Antlion }_{j}^{t}+c^{t}, \\
& d_{i}^{t}=\text { Antlion }_{j}^{t}+d^{t},
\end{aligned}
$$

where antlion ${ }_{j}^{t}$ is the position of the $j$ th ant lion at $t$ th iteration and $d^{t}$ and $c^{t}$ are the upper and lower bounds of all variables at $t$ th iteration, respectively, which can be represented by Equation (6) and Equation (7):

$$
\begin{aligned}
& c^{t}=\frac{c^{t}}{10^{w}(t / T)}, \\
& d^{t}=\frac{d^{t}}{10^{w}(t / T)},
\end{aligned}
$$

where $t$ is the current iteration, $T$ is the largest iteration, and $w$ is a constant determined by $T$ and $t$.

The best ant lion is selected as the elite ant lion, and the roulette selection ant lion is used to guide the ant position update; it can be expressed as follows:

$$
\operatorname{Ant}_{i}^{t}=\frac{R_{A}^{t}+R_{E}^{t}}{2}
$$

The next step is to update the archive and deal with the situation of exceeding the archive capacity. The archive is the Pareto optimal solution set, which stores the ant's position and fitness value in the Pareto optimal solution set. The archive needs to be updated after each update of ant's position and fitness value in the iteration process. When archive is full, the solutions with most populated neighborhood are removed from the archive to accommodate new solutions. The probability of removal is expressed as follows:

$$
P_{i}=\frac{N_{i}}{c}
$$


If the ant falls to the bottom of the trap and is caught by the ant lion, the individual will be regarded as a new ant lion to rebuild the trap if the population contains an individual with higher fitness than the ant lion; it can be defined by Equation (10):

$$
\operatorname{Antlion}_{j}^{t}=\operatorname{Ant}_{i}^{t}, \text { if } f\left(\operatorname{Ant}_{i}^{t}\right)>f\left(\operatorname{Antlion}_{j}^{t}\right),
$$

where $R_{A}{ }^{t}$ is the random walk around the antlion selected by the roulette wheel at $t$ th iteration, $R_{E}{ }^{t}$ is the random walk around the elite at $t$ th iteration, and $\mathrm{Ant}_{i}{ }^{t}$ indicates the position of $i$ th ant at $t$ th iteration.

3.2. Improved MOALO Algorithm. In this section, we proposed an improved version of the MOALO algorithm (IMOALO) using levy flight. Levy flight strategy is used in PSO algorithm [25], which can prevent the decrease of population diversity and has good global search ability. Therefore, the combination of levy flight strategy and MOALO can effectively prevent it from falling into local optimum. The calculation formula of the step length $S$ of Mantegna's algorithm simulating Levy flight is as follows:

$$
S=\frac{u}{|v|^{1 / \beta}}
$$

where $\beta$ is referred to as Levy index, where $u$ and $v$ are drawn from normal distributions, the definition are as follows:

$$
\left\{\begin{array}{l}
u \sim N\left(0, \sigma_{u}^{2}\right), \\
v \sim N\left(0, \sigma_{v}^{2}\right),
\end{array}\right.
$$

where

$$
\sigma_{u}=\left\{\frac{\Gamma(1+\beta) \sin (\pi \beta / 2)}{\beta \Gamma[1+\beta / 2] 2^{(\beta-1) / 2}}\right\}^{1 / \beta}, \quad \sigma_{v}=1
$$

where $\Gamma$ is the gamma function, then the step size is calculated by

$$
S t=\text { scale } \times S .
$$

Here, scale is an adjustable factor, which can be adjusted according to different problems or iteration process, so as to avoid flying step too large or too small and try to keep in an appropriate range for efficient search.

Therefore, the population renewal formula with levy flight is as follows:

$$
\mathrm{pop}_{\text {new }}=\mathrm{pop}+r * S t
$$

where $r$ is a random number between $[0,1]$.

The pseudocodes of the improved MOALO algorithm 1 is as follows.

\section{Integrated Optimization of Pipe Routing and Clamp Layout Based on Improved MOALO}

4.1. Clamp-Based Path Mode and Clamp Coordinate Transform. Due to the existence of assembly constraints between pipe and clamp, inspired by engineering and expert experience, this paper proposes to establish a routing path model based on clamp constraint. As shown in Figure 4, the pipe path nodes $\left(P_{\mathrm{s}}, P_{\mathrm{t}}, P_{\mathrm{cl}}, P_{\mathrm{c} 2}\right)$ are composed of the extension point of the pipe connector and the extension point of the axis in the clamp. In order to make the pipe path mode have stronger ability of obstacle avoidance, the regular nodes $\left(P_{1}, P_{2}\right)$ can be inserted between the path nodes. In order to reduce the search space, the regular nodes are set in a certain distance from the casing surface.

Obviously, the path node is based on the design mode of clamp layout. Its advantage is that it can ensure that the clamp and the pipe meet the assembly constraint relationship and can be fixed on the clamp base accurately. At the same time, it can provide support for solving the coupling problem of pipe routing and clamp layout design.

In addition, considering the machinability and reducing the flow resistance of the pipe, arc processing is carried out at the bending position of the pipe, as shown in Figure 5, where point $O$ is the center coordinate of the $\operatorname{arc} ; R$ is the bending radius, and it has certain empirical mapping relationship with the pipe diameter.

This paper takes the clamp based on the mounting for example. Since the clamp needs to be fixed on the surface of the casing, the normal direction of the clamp base changes with the position of the mounting point. In order to calculate the clamp position, a relative coordinate system $\left\{O^{\prime}, e_{1}{ }^{\prime}, e_{2}{ }^{\prime}\right.$ ,$\left.e_{3}{ }^{\prime}\right\}$ is constructed, where $e_{1}{ }^{\prime}, e_{2}{ }^{\prime}$, and $e_{3}{ }^{\prime}$ are unit vectors that satisfy the right-handed relationship, $O^{\prime}$ is the origin point, $e_{3}$ ' is the axis direction of the clamp base. The geometric coordinates of the clamp are calculated in this coordinate system. Finally, the coordinates are transformed into the absolute coordinate system $\left\{O, e_{1}, e_{2}, e_{3}\right\}$ by affine transformation.

Suppose $(x, y, z)$ and $\left(x^{\prime}, y^{\prime}, z^{\prime}\right)$ are the coordinates of a point in $\left\{O, e_{1}, e_{2}, e_{3}\right\}$ and $\left\{O^{\prime}, e_{1}^{\prime}, e_{2}{ }^{\prime}, e_{3}^{\prime}\right\}$, respectively, the relationship between them can be expressed by affine transformation in Equation (16):

$$
\left(\begin{array}{l}
x \\
y \\
z
\end{array}\right)=\left(\begin{array}{lll}
a_{11} & a_{12} & a_{13} \\
a_{21} & a_{22} & a_{23} \\
a_{31} & a_{32} & a_{33}
\end{array}\right)\left(\begin{array}{c}
x^{\prime} \\
y^{\prime} \\
z^{\prime}
\end{array}\right)+\left(\begin{array}{l}
x_{0} \\
y_{0} \\
z_{0}
\end{array}\right),
$$

where matrix $A=\left(a_{i j}\right)$ is the transition matrix, which satisfies:

$$
\left(e_{1}^{\prime}, e_{2}^{\prime}, e_{3}^{\prime}\right)=\left(e_{1}, e_{2}, e_{3}\right)\left(\begin{array}{lll}
a_{11} & a_{12} & a_{13} \\
a_{21} & a_{22} & a_{23} \\
a_{31} & a_{32} & a_{33}
\end{array}\right) .
$$




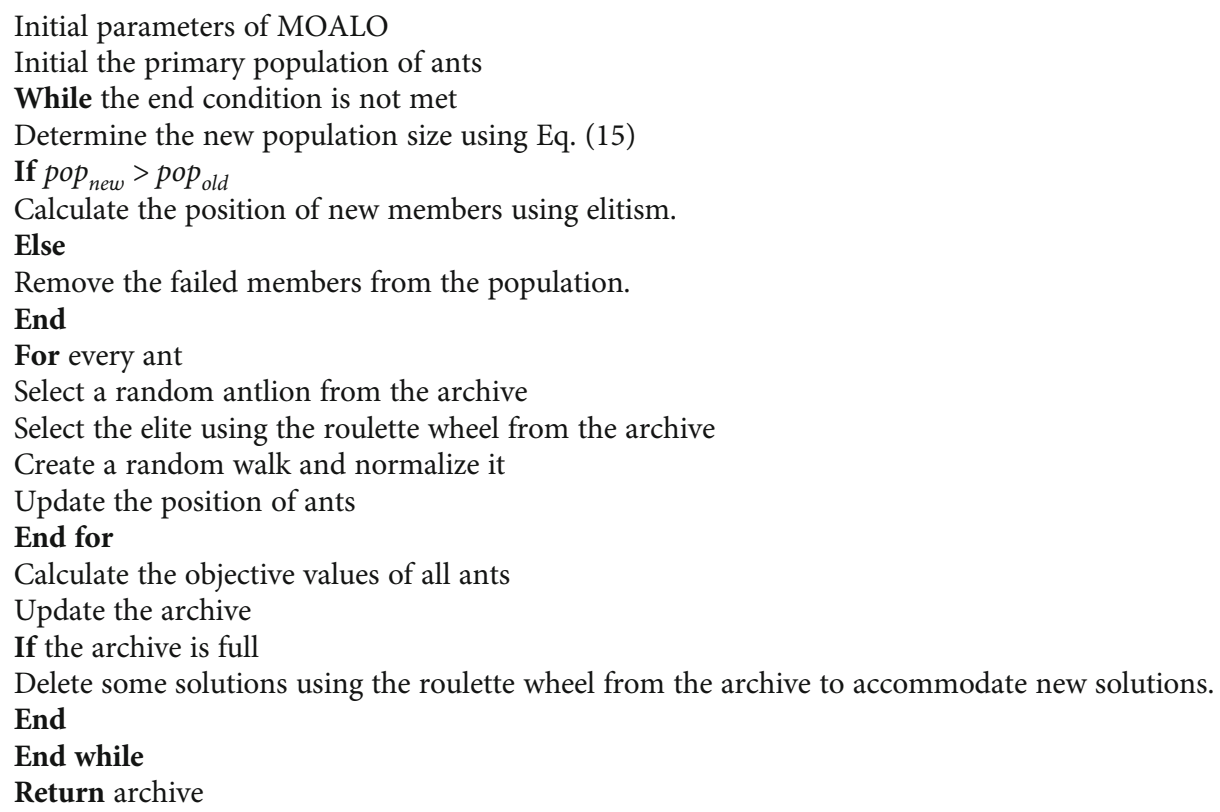

Algorithm 1: The improved MOALO.

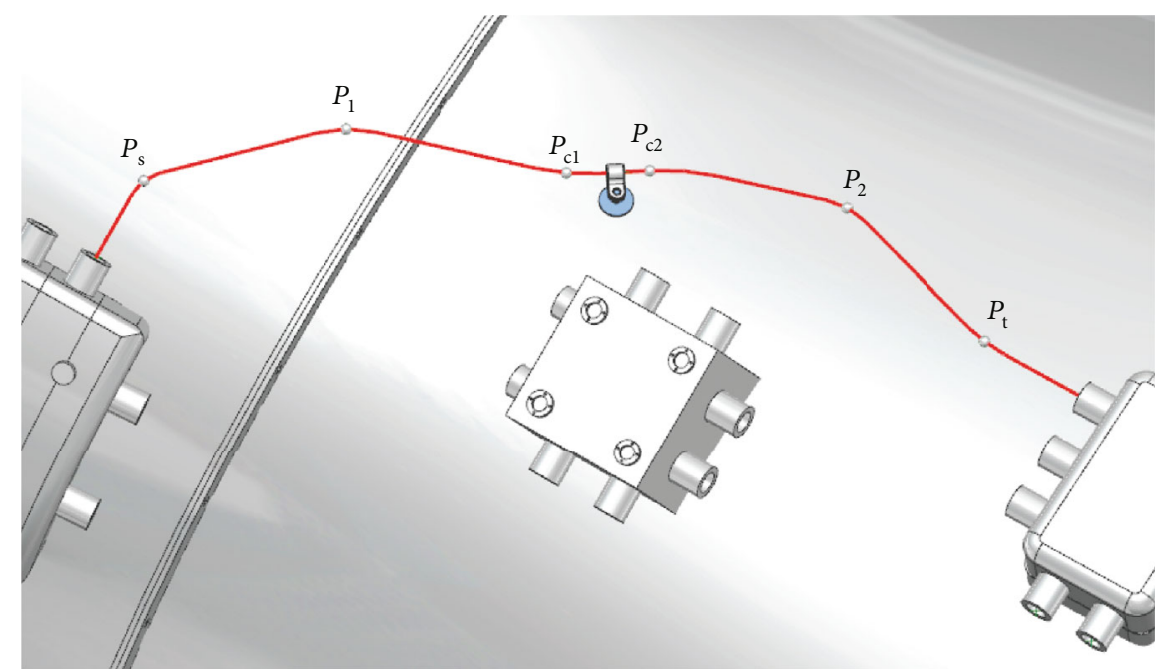

FIgURE 4: Clamp-based pipe path mode.

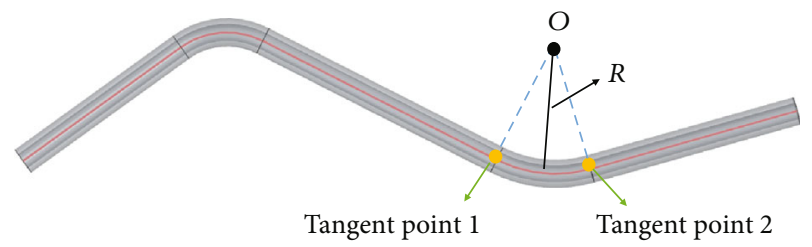

FIGURE 5: Bending radius of pipe.

4.2. Individual Encoding Design. The decision variables of pipe routing and clamp layout are coded as a whole and adopts fixed length coding to facilitate the design and implementation of the algorithm. In practical application, the number of clamps is 0-2. As shown in Figure 6, it takes the clamp position coordinate $\left(O^{\prime}\right)$, clamp rotation angle $(\theta)$, and extension length at both ends of the clamp point $(K)$ as codes. At the same time, the regular nodes of the path are coded. In summary, the specific coding forms are as follows in Figure 7.

It should be noted that since the center point of the clamp base is located on the surface of the casing, and the surface equation of the casing can be obtained in 2.4, the twodimensional coding of the center point of the clamp base can describe its three-dimensional position. Furthermore, the normal direction of the clamp base can be solved by the space geometry method and the surface equation of the casing. In order to calculate the regular node, according to the coding information to get the point coordinate of the casing 


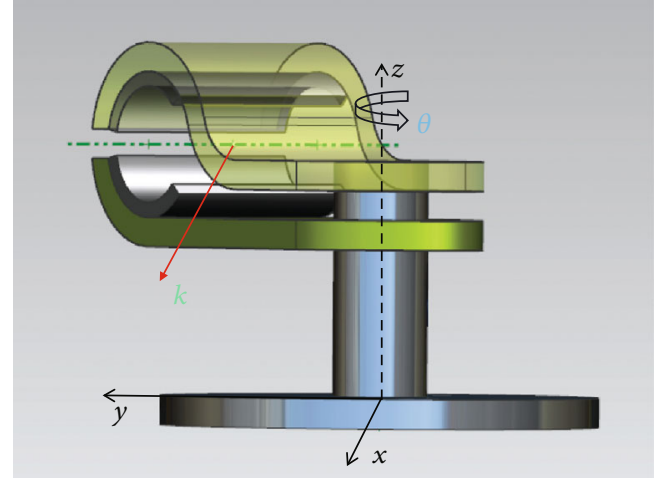

Figure 6: Calculation of clamp posture.

surface first, and then, a distance is extended from the normal direction of the point, which is the coordinates of the regular nodes.

4.3. Objective Functions and Constraint Processing. This paper takes pipe length $f_{L}$ and pipe smoothness (bend angle) $f_{\mathrm{C}}$ as optimization objectives. $f_{L}$ and $f_{\mathrm{C}}$ can be calculated by Equations (18) and (19).

$$
\begin{aligned}
& f_{\mathrm{L}}=\sum_{i=1}^{m-1} \sqrt{\left(x_{i+1}-x_{i}\right)^{2}+\left(y_{i+1}-y_{i}\right)^{2}+\left(z_{i+1}-z_{i}\right)^{2}}, \\
& f_{\mathrm{C}}=\sum_{i=1}^{m-2} \beta_{i}=\sum_{i=1}^{m-2} \arccos \left(\vec{a}_{i} \cdot \vec{b}_{i}|| \vec{a}_{i}|\cdot| \vec{b}_{i} \mid\right),
\end{aligned}
$$

where $m$ is the number of nodes in the path; $\left(x_{i}, y_{i}, z_{i}\right)$ is the node coordinate of the path; $\vec{a}_{i}=\left(x_{i+1}-x_{i}, y_{i+1}-y_{i}, z_{i+1}-\right.$ $\left.z_{i}\right), \vec{b}_{i}=\left(x_{i+2}-x_{i+1}, y_{i+2}-y_{i+1}, z_{i+2}-z_{i+1}\right)$.

It should be noted that the objective functions will be converted into a minimum value for calculation, so formula (14) is the complementary angle of pipe angle.

For pipe routing constraints (1-3), penalty function method is used to deal with it; for pipe constraint (4), the algorithm solves the problem by limiting the distance between regular node and casing surface; for pipe constraint (5), the corresponding table of pipe diameter and bending radius will be established to solve it.

For the clamp layout constraint (1), it will be limited in the designed area during the population initialization. If the clamp deviates from the designed area in the iteration process, the algorithm will automatically adjust its position; for the clamp constraint (2), penalty function method is used to deal with it; for the clamp constraint (3), the "clampbased path mode" established in 4.1 is adopted to solve it.

Considering the above factors, the objective functions considering the penalty term can be expressed using Equations (20) and (21).

$$
\begin{aligned}
& f_{1}=f_{\mathrm{L}}+\alpha \cdot k_{\mathrm{P}}+\beta \cdot k_{\mathrm{C}}, \\
& f_{2}=f_{\mathrm{C}}+\alpha \cdot k_{\mathrm{P}}+\beta \cdot k_{\mathrm{C}},
\end{aligned}
$$

where $K_{\mathrm{P}}$ and $K_{\mathrm{C}}$ are the punishment terms corresponding to the pipe routing and the clamp layout, respectively, which are larger positive numbers; $\alpha$ and $\beta$ are the coefficient of penalty term, and the value is 1 when the penalty is generated; otherwise, it is 0 .

4.4. Routing System and Algorithm Flowchart. The routing system is constructed by using customized developments of MATLAB and Siemens NX systems, where data are transferred among these systems via TXT plain data files. More specially, in the aspect of modeling and preprocessing of routing model, the grid information of CAD model is extracted by the secondary development tool of Siemens/GRIP, and the TXT text is used as the input of algorithm. In order to speed up the operation, the TXT text data is converted to MAT format in MATLAB. In terms of routing algorithm, it is programmed in MATLAB platform to automatically calculate the pipe-clamp layouts, and the calculation results are saved as TXT text. Finally, the calculation results are returned to Siemens NX through GRIP secondary development program to realize the visualization of layout results. The overall flowchart of the presented routing optimization method is shown in Figure 8.

\section{Numerical Computations and Routing Examples}

5.1. Numerical Computation. The improved MOALO algorithm is tested and compared with the well-regarded multiobjective algorithm MOPSO and standard MOALO. The test functions are commonly used ZDT1 ZDT4, and GD (Generational Distance) is used as the metrics to measure the advantages and disadvantages of the two algorithms. Each algorithm runs 20 times, and the average value, variance, optimal value, and worst value of GD are calculated. The comparison data are shown in Table 1.

It can be seen from the above table that, as far as these test functions are concerned, the average value, variance, optimal value, and worst value of MOALO are better than MOPSO and standard MOALO. Therefore, this paper used the improved MOALO algorithm to optimize the layout of aeroengine pipes and clamps.

5.2. Routing Examples. The pipe routing and clamp layout algorithm is implemented on computer (Intel(R) CPU i7$6700 @ 3.40 \mathrm{GHz}, 8 \mathrm{~GB}$ RAM), with customized developments of MATLAB and Siemens NX systems.

In this paper, the layout of pipes and clamps are optimized by using IMOALO, MOALO, and MOPSO under the same conditions. The parameters of IMOALO and MOALO are set as follows: population size $=30$, iteration times $=30$, and the archive size $=15$. The parameters of MOPSO are set as follows: population size $=30$, iteration times $=30, c_{1}=c_{2}=1.4962, w=0.7298, \gamma=2$, and $\beta=2$. Figure 9 shows the distribution of representative Pareto solutions of the three algorithms.

It can be seen from the figure above that the solution effect of IMOALO is better than MOALO and MOPSO according the Pareto optimal front. The eight Pareto 


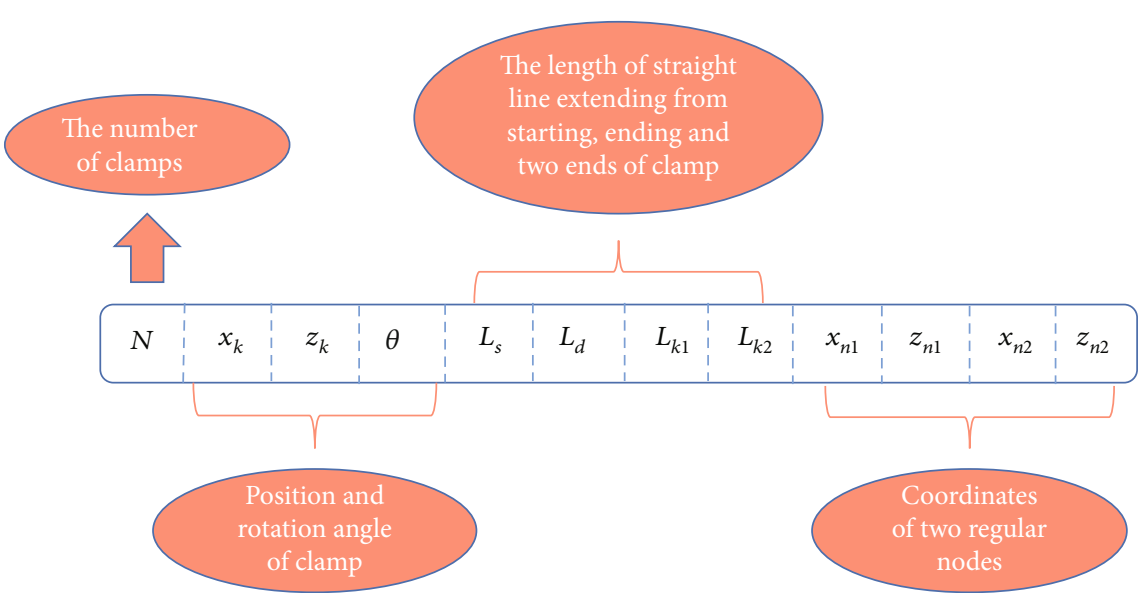

FIgURE 7: Individual coding form.

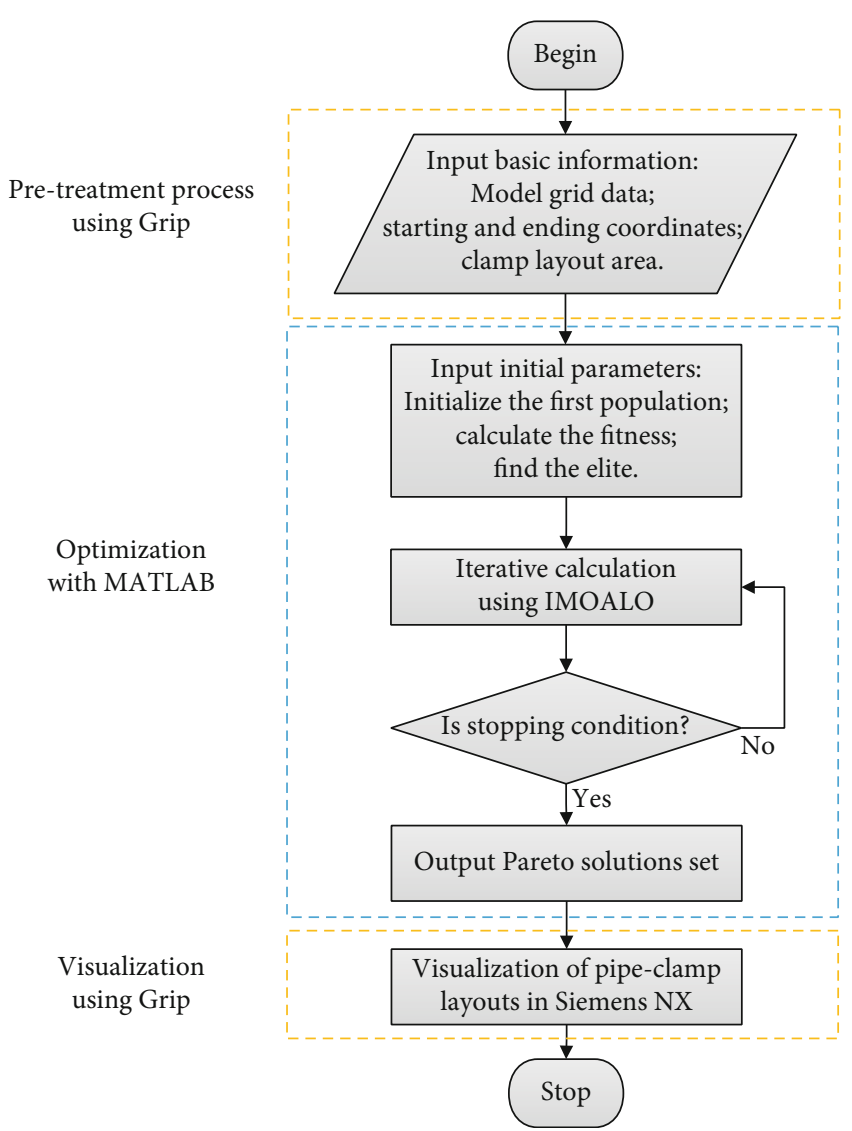

Figure 8: The flowchart of layout method.

solutions of IMOALO satisfy the constraint conditions and are evenly distributed in the target space. Taking one clamp as example, the routing results of three representative nondominated solutions on a simplified routing model are shown in Figure 10.

The above results show that the method can obtain collision-free pipe paths and appropriate clamp layouts while meeting certain engineering rules. In addition, pipe, clamp, and casing satisfied the assembly constraints as well. The obtained nondominated solutions provide designers with a
TABLE 1: Results of the multiobjective algorithms (using GD).

\begin{tabular}{lcccc}
\hline & Ave & Var & Best & Worst \\
\hline Algorithm ZDT1 & & & & \\
MOPSO & 0.005087 & $2.0547 E-6$ & 0.003160 & 0.008830 \\
MOALO & 0.002974 & $1.1426 E-6$ & 0.000533 & 0.004710 \\
IMOALO & 0.001184 & $2.8685 E-7$ & 0.000498 & 0.002313 \\
Algorithm ZDT2 & & & & \\
MOPSO & 0.003591 & $1.5219 E-6$ & 0.000209 & 0.006501 \\
MOALO & 0.002300 & $7.6867 E-7$ & 0.000920 & 0.004878 \\
IMOALO & 0.000848 & $2.4213 E-7$ & 0.000196 & 0.001811 \\
Algorithm ZDT3 & & & & \\
MOPSO & 0.007008 & $1.8337 E-5$ & 0.003432 & 0.020274 \\
MOALO & 0.005580 & $8.4764 E-6$ & 0.000458 & 0.010607 \\
IMOALO & 0.002231 & $2.0542 E-6$ & 0.000465 & 0.004713 \\
Algorithm ZDT4 & & & & \\
MOPSO & 0.008441 & $1.6125 E-4$ & 0.003677 & 0.062375 \\
MOALO & 0.002450 & $1.9174 E-6$ & 0.000342 & 0.006357 \\
IMOALO & 0.000883 & $1.7624 E-7$ & 0.000231 & 0.001888 \\
\hline
\end{tabular}

variety of choices to meet the preferences and experience in engineering practice. Compared with existing pipe routing methods, such as $[16,26]$, the presented method not only solves the pipe routing but also solves the clamp layout problems.

Finally, in order to further verify the engineering application value of the algorithm, this paper optimizes the layout of 16 pipes in sequence (as shown in Figure 11). After the optimization of each pipe is completed, the algorithm automatically performs obstacle processing on it. The total running time is about 86.72 seconds, averaging about 5.42 seconds for routing one pipe.

\section{Conclusion}

In this paper, the design coupling and assembly constraint relationship between pipe routing and clamp layout are discussed, and a pipe routing method considering clamp 


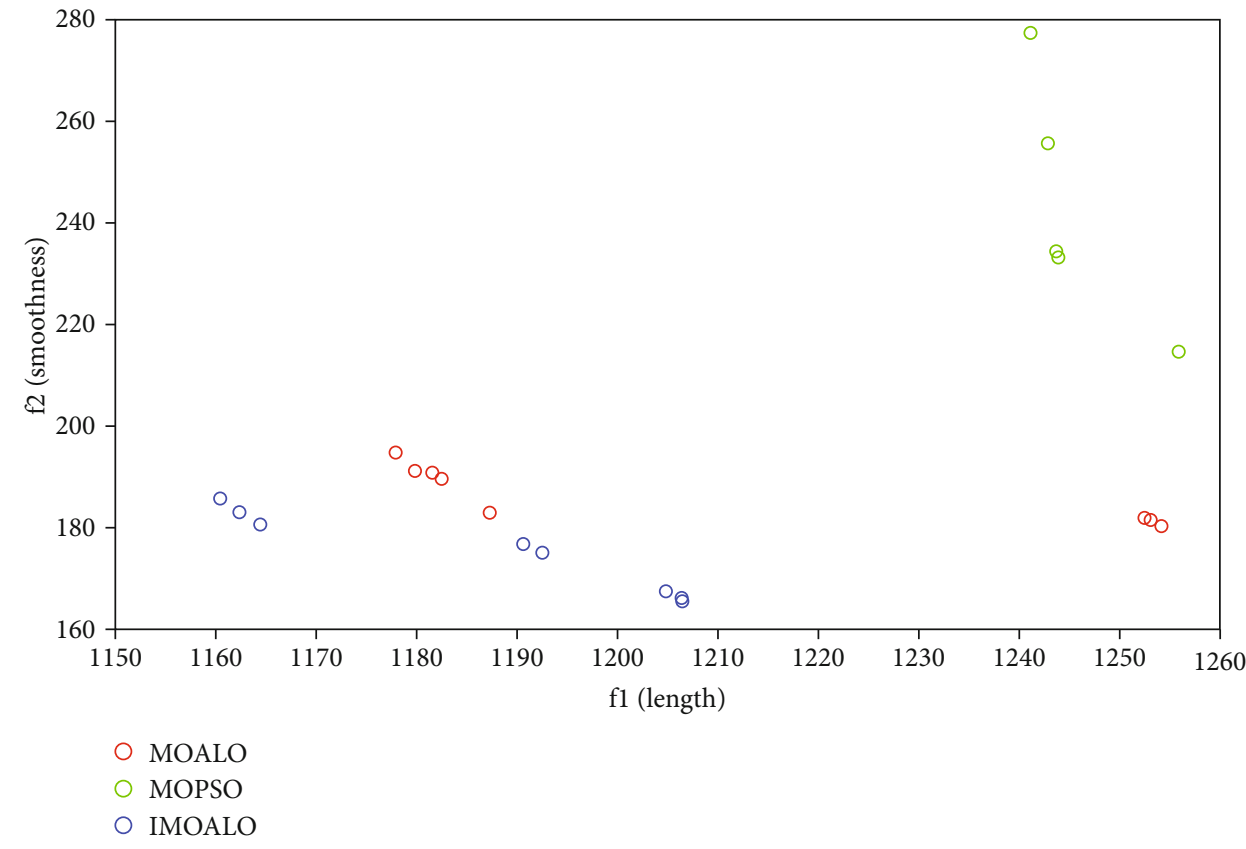

Figure 9: Pareto solution distribution.

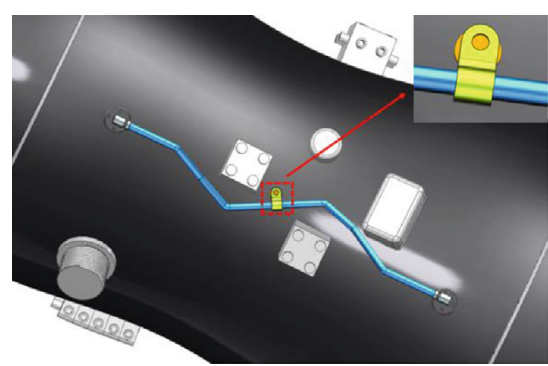

(a) Nondominated solution 1

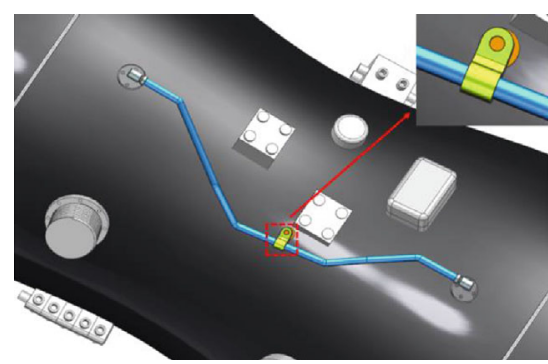

(b) Nondominated solution 2

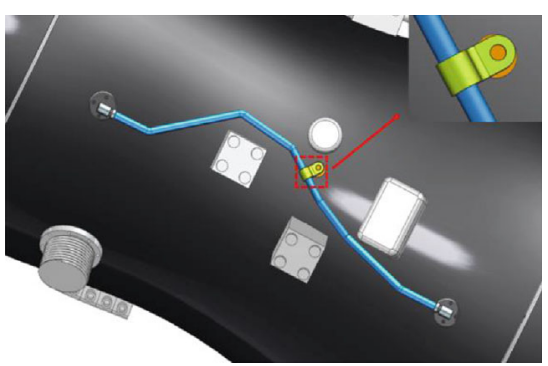

(c) Nondominated solution 3

FIgURE 10: Visualization of routing example.

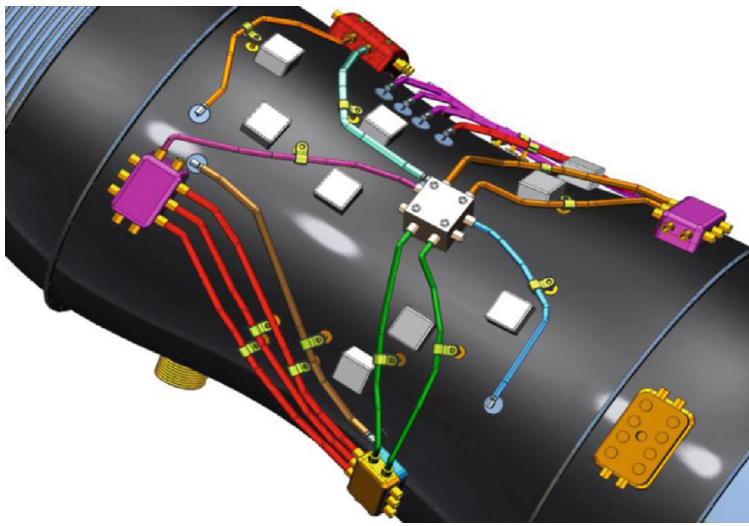

(a) View 1

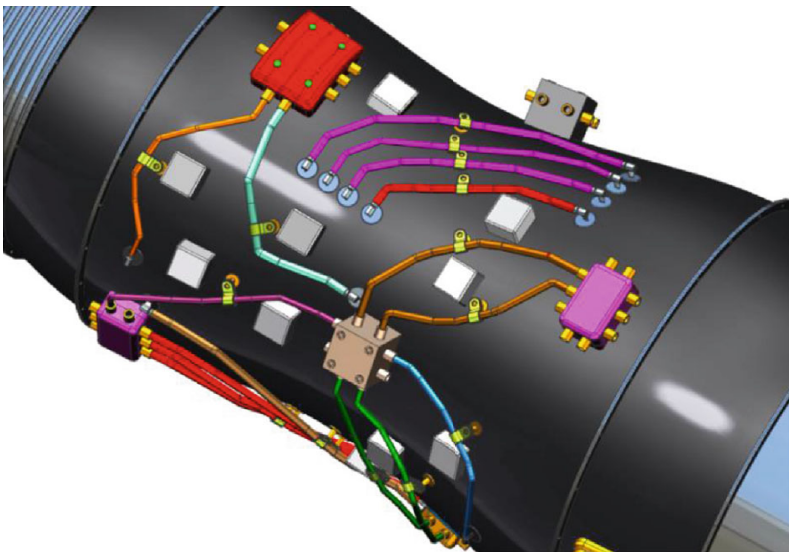

(b) View 2

FIGURE 11: Visualization of multipipes routing example.

constraints is presented by using improved MOALO. In particular, a clamp-based path mode is established, where the posture transformation calculation of clamp model is further given by using affine transformation method. The individual encoding, objective functions, and constraint processing method are given in detail.

Compared with the traditional pipe routing methods, the presented routing method comprehensively considers the 
relevant engineering rules of pipe routing and clamp layout and realizes the integrated optimization and automatic calculation of pipe path, clamp geometric position, and spatial posture parameters. The final routing examples demonstrate the presented routing method. In terms of considering the clamp layouts, the vibration performance is primarily considered from the perspective of geometric layout based on expert experience. Further study should be on extending the routing method to consider the pipe natural frequency and other types of clamps.

\section{Data Availability}

No data were used to support this study.

\section{Conflicts of Interest}

The authors declare that there is no conflict of interest regarding the publication of this paper.

\section{Acknowledgments}

This study was supported in part by Major Basic Research Projects of Aeroengine and Gas Turbine (No. J2019-I-00080008) and Key Projects of Liaoning Provincial Natural Science Foundation (No. 20170540589).

\section{References}

[1] H. T. Sui and W. T. Niu, "Branch-pipe-routing approach for ships using improved genetic algorithm," Frontiers of Mechanical Engineering, vol. 11, no. 3, pp. 316-323, 2016.

[2] C. E. Wang and Q. Liu, "Projection and geodesic-based pipe routing algorithm," IEEE Transactions on Automation Science and Engineering, vol. 8, no. 3, pp. 641-645, 2011.

[3] B. X. Zhao, J. H. Liu, and R. X. Ning, "An automatic pipeline layout and comprehensive optimization technology based on engineering constraints," Journal of Mechanical Engineering, vol. 51, no. 21, pp. 121-131, 2015.

[4] Y. Chen, T. Li, and M. A. Yin, "Research on pipeline automatic laying based on improved A * algorithm," Journal of Ordnance Equipment Engineering, vol. 39, no. 10, pp. 91-95 $+104,2018$.

[5] C. van der Velden, C. Bil, X. H. Yu, and A. Smith, "An intelligent system for automatic layout routing in aerospace design," Innovations in Systems and Software Engineering, vol. 3, no. 2, pp. 117-128, 2007.

[6] J. Ma, J. Liu, L. Xu, H. Wu, and J. Xu, "Method based on LTLPRM algorithm," Journal of Mechanical Engineering, vol. 54, no. 15 , pp. 160-170, 2018.

[7] Q. Liu and G. S. Jiao, "Multi objective layout optimization of aeroengine pipeline based on improved NSGA-II," Computer Integrated Manufacturing Systems, vol. 24, no. 5, pp. 12171227, 2018.

[8] D. Baeza, C. F. Ihle, and J. M. Ortiz, "A comparison between ACO and Dijkstra algorithms for optimal ore concentrate pipeline routing," Journal of Cleaner Production, vol. 144, pp. 149-160, 2017.

[9] J. G. Min, W. S. Ruy, and C. S. Park, "Faster pipe auto-routing using improved jump point search," International Journal of
Naval Architecture and Ocean Engineering, vol. 12, pp. 596$604,2020$.

[10] W. Y. Jiang, Y. Lin, M. Chen, and Y. Y. Yu, "A co-evolutionary improved multi-ant colony optimization for ship multiple and branch pipe route design," Ocean Engineering, vol. 102, pp. 63-70, 2015.

[11] Q. Zhou and Y. J. Lv, "Research based on lee algorithm and genetic algorithm of the automatic external pipe routing of the aircraft engine," International Journal of Mechanical Engineering and Applications, vol. 8, no. 1, pp. 40-44, 2020.

[12] Z. R. Dong and Y. Lin, "A particle swarm optimization based approach for ship pipe route design," International Shipbuilding Progress, vol. 63, no. 1-2, pp. 59-84, 2017.

[13] Y. L. Wang, Y. Y. Yu, K. Li, X. G. Zhao, and G. Guan, “A human-computer cooperation improved ant colony optimization for ship pipe route design," Ocean Engineering, vol. 150, pp. 12-20, 2018.

[14] Y. F. Qu, D. Jiang, and Q. Y. Yang, "Branch pipe routing based on $3 \mathrm{D}$ connection graph and concurrent ant colony optimization algorithm," Journal of Intelligent Manufacturing, vol. 29, no. 7, pp. 1647-1657, 2018.

[15] S. Haiteng, N. Wentie, N. Yaxiao, Z. Chongkai, and G. Weigao, "Pipe-assembly approach for ships using modified NSGA-II algorithm," Computer Aided Drafting, Design and Manufacturing, vol. 26, no. 2, pp. 34-42, 2016.

[16] Q. Liu and G. S. Jiao, "A pipe routing method considering vibration for aeroengine using kriging model and NSGA-II," IEEE Access, vol. 6, pp. 6286-6292, 2018.

[17] F. Ahmed and K. Deb, "Multi-objective optimal path planning using elitist non-dominated sorting genetic algorithms," Soft Computing, vol. 17, no. 7, pp. 1283-1299, 2013.

[18] Z. G. Jia and Z. Y. Chen, "Research on the method of aeroengine pipeline frequency modulation based on parameterization," Aeroengine, vol. 34, no. 4, pp. 34-37, 2008.

[19] Y. Q. Chen and Z. G. Zhu, "Optimization design of aeroengine pipeline based on genetic algorithm," Journal of Aerospace Power, vol. 4, pp. 421-425, 2002.

[20] J. H. Lv, C. P. Zang, R. W. Zhang, G. B. Zhang, and X. W. Wang, "Nonlinear equivalent modeling method of clamp based on test data," Journal of Aerospace Power, vol. 34, no. 9, pp. 1944-1952, 2019.

[21] P. Gao, J. Li, J. Zhai, Y. Tao, and Q. Han, “A novel optimization layout method for clamps in a pipeline system," Applied Sciences, vol. 10, no. 1, p. 390, 2020.

[22] Q. Liu and G. S. Jiao, "Optimization of aeroengine pipeline clamp layout based on Kriging model and NSGA-II," CAAI Transactions on Intelligent Systems, vol. 14, no. 2, pp. 281287, 2019.

[23] S. Mirjalili, P. Jangir, and S. Saremi, "Multi-objective ant lion optimizer: a multi-objective optimization algorithm for solving engineering problems," Applied Intelligence, vol. 46, no. 1, pp. 79-95, 2017.

[24] S. Mirjalili, "The ant lion optimizer," Advances in Engineering Software, vol. 83, pp. 80-98, 2015.

[25] H. Haklı and H. Uğuz, "A novel particle swarm optimization algorithm with Levy flight," Applied Soft Computing, vol. 23, pp. 333-345, 2014.

[26] J. P. Yu, H. X. Yuan, Y. H. Yang, S. Zhang, and Q. Fei, “Aeroengine pipe layout optimization based on adaptive beetle antennae search algorithm," Journal of Mechanical Engineering, vol. 56, no. 20, p. 174, 2020. 